\title{
THE EFFECTS OF TIME DEPENDANT CONVECTION ON WHITE DWARF RADIAL PULSATIONS
}

\author{
S. Starrfield \\ Theoretical Division, T-6, MSB288 \\ Los Alamos National Laboratory, Los Alamos, NM 87545 \\ and Department of Physics Arizona State University, Tempe, AZ 85287 \\ and \\ A. N. Cox \\ Theoretical Division, T-6, MS B288 \\ Los Alamos National Laboratory, Los Alamos, N.M. 87545
}

\begin{abstract}
We have investigated the effects of relaxing the normal assumption of frozen in convection on studies of radial instabilities in $0.6 \mathrm{M}_{\odot}$ carbon-oxygen white dwarfs with either pure hydrogen layers overlying pure helium layers or $0.6 \mathrm{M}_{\odot}$ carbon-oxygen white dwarfs with pure helium surface layers. In this paper we assume that convection can adjust to the pulsation at a rate determined by the time scale of a convective eddy. Using this assumption in our analysis stabilizes most of the modes in both the DA and DB radial instability strips. We also find that the blue edge of the DA radial instability strip, assuming frozen in convection, is between $12,000 \mathrm{~K}$ and $13,000 \mathrm{~K}$. The blue edge for the DB radial instability strip (frozen in convection) is between $32,000 \mathrm{~K}$ and $33,000 \mathrm{~K}$.
\end{abstract}

\section{INTRODUCTION}

The first member of the $\mathrm{ZZ}$ Ceti class of nonradially pulsating white dwarfs, HL Tau 78, was discovered by Arlo Landolt in 1964. Since its periods and those of other members of the class, discovered later, were much longer than the radial pulsation periods of white dwarfs, it was proposed by both Chanmugam (1972) and Warner and Robinson (1972) that these stars were pulsating in nonradial $g^{+}$modes.

However, the first discovery of an excitation mechanism in DA dwarfs was found for the radial modes of very short period (Cox, Hodson, and Starrfield 1980). In this case the driving was the normal $\kappa$ and $\gamma$ effects that are well known from studies of Cepheids and RR Lyrae variables. At that time we did not have a nonradial analysis code available to us and were unable to test our stellar models for nonradial instabilities. This problem was solved by Winget, Van Horn, and Hansen (1981) who used the Saio and Cox (1980) method to analyze both DA and DB dwarfs for instability and found that they were unstable in the same $T_{e}$ range that we had found radial instabilities.

After that time much of the theoretical work on the DA and DB instability strips was done in order to understand the cause of the driving in the nonradial $g^{+}$modes which are observed in these stars (recent reviews can be found in Starrfield 1987; Winget 1988).

Further work on the radial instability was done by Saio, Winget, and Robinson (1983), Starrfield et al.(1983), and Cox et al.(1987; hereafter CSKP). In all cases they found that low order radial modes were unstable both in the DA and the DB nonradial instability strips. However, none of the observational studies of either these variables or stars close, in $T_{e}$, to these variables found any sign of a radial instability (Robinson 1984; and references therein). 
This is an important confrontation between theory and observations since the same white dwarf models are used both in the radial and nonradial studies and, in addition, the same radial analysis methods are used in studies of Cepheids and RR Lyr variables where they have proved to be very successful. Therefore, we have continued to search for a physical mechanism that could damp out the radial instability but would not completely stabilize the nonradial modes.

\section{METHOD OF ANALYSIS AND IMPORTANCE OF CONVECTION}

One such mechanism is the interaction between pulsation and convection. In our nonradial analyses of the DAV (ZZ Ceti) and DBV classes of variable stars (CSKP), we identified and described the importance of a new driving mechanism for stellar pulsations: convection blocking. This mechanism depends upon the fact that a surface convective region cannot react instantaneously to a change in the energy flow from pulsation and when it carries most of the energy it can block the changing flow of radiation from below and drive pulsations analogous to the $\kappa$-effect. This pulsation-convection interaction must be occurring since the pulsational motion of the zones changes the temperature gradient within the convective region so that the flow of energy (through the region) will vary in a very complex fashion. Time dependant convection (TDC) will actually drive pulsations if the period of pulsation is shorter than the convective eddy time scale. If the pulsation period is of the same order or longer than the convective eddy time scale, then convection should damp pulsations.

In an attempt to simulate TDC, we have now added the method of Cox, Brownlee, and Eilers (1966; see also Cox and Giuli 1968) to the Los Alamos linear, nonadiabatic, analysis (LNA) code (CSKP). Their technique removes the assumption of frozen-in convection (the normal assumption in both the radial and nonradial studies of white dwarfs) but also assumes that convection cannot adjust instantaneously to a perturbation. There will be some lag in the adjustment which depends on the ratio of the convective eddy time scale to the pulsation period. They write the perturbation in the convective flux in terms of a constant part plus a time varying part which is assumed to exhibit sinusoidal variations. When this technique is included, it is found that if the pulsation period is much shorter than the convective eddy time scale (the mixing length divided by the convective velocity in the Böhm-Vitense 1958 theory), it becomes difficult for the convective region to adjust to the pulsation and the flow of energy allowed by convection lags the pulsation. In the most extreme case we again find convection blocking and the lag is sufficient to drive rather than damp the instability. Here, we are concerned mostly with a lag that is almost 90 degrees out of phase with the pulsation. Finally, we note that we cannot add the procedure as described by Cox, Brownlee, and Eilers (1966) directly into our nonradial analysis code because it is unable to specify the amount of horizontal energy flow.

In the analysis reported in this paper we use the above LNA code with both TDC and non-TDC (NTDC). We have described this program and our models in some detail elsewhere (CSKP) and will not include that discussion here. We searched in $\mathrm{T}_{e}$ from $9000 \mathrm{~K}$ to $14,000 \mathrm{~K}$ for the DA radial instability strip and from $24,000 \mathrm{~K}$ to $35,000 \mathrm{~K}$ for the DB radial instability strip. We use various values of $1 / H_{p}$ and find that, as expected, our hottest blue edges require efficient convection (Fontaine, Tassoul, and Wesemael 1984).

\section{RESULTS AND DISCUSSION}

Our principle result is that for most of the unstable modes found in studies with NTDC, the assumption of TDC stabilizes the modes. There are a few fundamental or very high order modes 

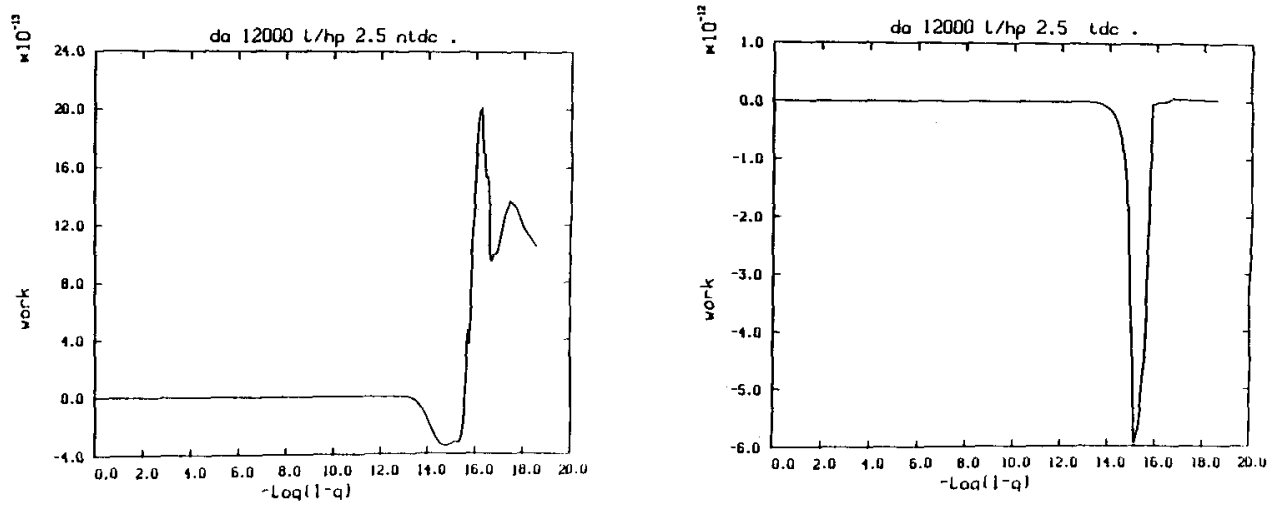

Figure 1a. This is the work plot for the ninth overtone in a DA dwarf with $\mathrm{T}_{e}=12,000 \mathrm{~K}, 1 / \mathrm{H}_{\mathrm{p}}$ $=2.5$, and frozen-in convection (NTDC) assumed. Peak driving occurs at the bottom of the convective region which has a temperature of $33,000 \mathrm{~K}$. The period of this mode is $1.43 \mathrm{~s}$ and it is unstable.

Figure $1 \mathrm{~b}$. This is the work plot for the same model in which time dependant convection (TDC) is assumed. All of the driving has disappeared and this mode is stable. Note the difference in scales of the abscissae between the two plots.
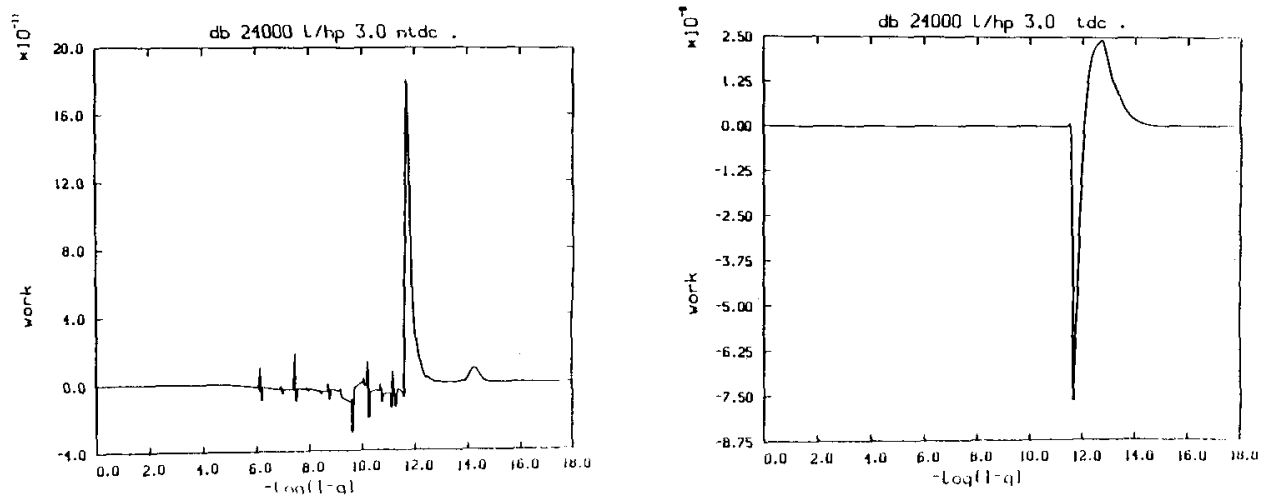

Figure 2a. This is the work plot for the ninth overtone in a DB dwarf with $\mathrm{T}_{e}=24,000 \mathrm{~K}, 1 / H_{p}$ $=3.0$, and frozen-in convection (NTDC) assumed. The sharp spikes occur at tabular points and the work sums to zero. The period of this mode is $1.34 \mathrm{~s}$, peak driving occurs at a depth where the temperature is $270,000 \mathrm{~K}$, and it is unstable.

Figure 2b. The work plot for the same model in which time dependant convection (TDC) is assumed. Again, note the difference in scales of the two abscissae. The masses of the zones increase inwards so that there is more damping than driving in this model and it is stable. 
where TDC causes an instability but this is because the deepest zone of the convective region has a large convective time scale. This driving may disappear when we include more zones.

We found that the analyses done with NTDC produced a DA radial instability strip that extended from below $9000 \mathrm{~K}$ (we did not search any cooler in $\mathrm{T}_{e}$ ) to $12,000 \mathrm{~K}$. The models at $13,000 \mathrm{~K}$ and $14,000 \mathrm{~K}$ were stable except for 3 very high order modes at 13,000 (TDC) that were probably driven by only one zone. The models with $\mathrm{l} / H_{p}=2.5$ were, in general, more unstable than the models with smaller values of $1 / h_{p}$. Figure 1 shows two work plots for the 9 th overtone in a $12,000 \mathrm{~K}$ model. The differences are obvious.

For the DB instability strip, we found that models with frozen in convection (NTDC) were unstable for $\mathrm{T}_{e}$ 's from 24,000 (we did not go cooler) to $32,000 \mathrm{~K}\left(1 / H_{p}=3.0\right.$ ). Models hotter than this were stable. We were surprised to find that the instability strip extends to this high an $T_{e}$ since the blue edge for the nonradial instability strip is at $\sim 27,000 \mathrm{~K}$. Figure 2 shows two work plots for the 9th overtone in a $24,000 \mathrm{~K}$ model and it is clear that TDC has stabilized this mode. Some modes at $33,000 \mathrm{~K}$ and $35,000 \mathrm{~K}$ were unstable when we analyzed the envelopes assuming TDC. As in the DA's, they seemed to be driven by the deepest convective zone layers.

We gratefully acknowledge discussions with W.D. Pesnell and E. M. Sion. S. Starrfield thanks G. Bell, S. Colgate, M. Henderson, and J. Norman for the hospitality of the Los Alamos National Laboratory and a generous allotment of computer time. This work was partially supported by NSF grant AST85-16173 to Arizona State University and by the DOE.

\section{REFERENCES}

Böhm-Vitense, E., Zs. Ap., 46, 108, (1958).

Chanmugam, G., Nature Phys. Sci., 236, 83, (1972).

Cox, A. N., Brownlee, R. R., and Eilers, D. D., Astrophys. J., 144, 1024 (1966).

Cox, A. N., Hodson, S. W., and Starrfield, S., in Nonradial and Nonlinear Stellar Pulsations, ed. H. A. Hill and W. Dziembowski, Berlin, Springer-Verlag, p. 453, (1980),

Cox, A. N., Starrfield, S., Kidman, R. B., and Pesnell, W. D., Astrophys. J., 317, 303, (1987).

Cox, J. P., and Giuli, R. T., Principles of Stellar Structure, New York, Gordon and Breach, (1968).

Fontaine, G., Tassoul, M., and Wesemael, F., in Proc. 25th Liége Internat. Astrophys. Coll., ed. A. Noels and M. Gabriel, p. 328, (1984).

Robinson, E. L. Astron. J., 89, 1732, (1984).

Saio, H., and Cox, J. P., Astrophys. J., 236, 549, (1980).

Saio, H., Winget, D. E., and Robinson, E. L., Astrophys. J., 265, 982, (1983).

Starrfield, S., in Stellar Pulsation: A Memorial to John P. Cox, ed. A. N. Cox, W. M. Sparks, and S. Starrfield, Berlin, Springer-Verlag, p. 332, (1987).

Starrfield, S., Cox, A. N., Hodson, S. W., and Clancy, S. P., Astrophys. J., 269, 645, (1983).

Warner, B., and Robinson, E. L., Nature Physical Science, 329, 2, (1972).

Winget, D. E., in Advances in Helio and Asteroseismology ed. J. Christensen-Dalsgaard and S. Frandsen, Dordrecht, Reidel, p. 305, (1988).

Winget, D. E., Van Horn, H. M., and Hansen, C. J., Astrophys. J. Letters, 245, L33, (1981). 